\title{
On the numerical solution of Volterra-Fredholm integral equations with logarithmic kernel using smoothing transformation
}

\author{
Chokri Chniti ${ }^{1}$, Sharefa Eisa Ali Alhazmi ${ }^{2 *}$ \\ ${ }^{1}$ Nabeul Engineering Preparatory Institute, University of Carthage, Tunisia. \\ ${ }^{2}$ Umm Al-Qura University College of Education for Girls at Al-Qunfudah, Mathematics department, Macca, KSA \\ *Corresponding author E-mail: cachniti@uqu.edu.sa
}

\begin{abstract}
Copyright (C)2015 Chniti and Alhazmi. This is an open access article distributed under the Creative Commons Attribution License, which permits unrestricted use, distribution, and reproduction in any medium, provided the original work is properly cited.
\end{abstract}

\begin{abstract}
A smoothing transformation, Legendre and Chebyshev collocation method are presented to solve numerically the Voltterra-Fredholm Integral Equations with Logarithmic Kernel. We transform the Volterra Fredholm integral equations to a system of Fredholm integral equations of the second kind, using a smoothing transformation to cancel the singularities in the kernel, a system Fredholm integral equation with smooth kernel is obtained and will be solved using Legendre and Chebyshev polynomials. This lead to a system of algebraic equations with Legendre or Chebychev coefficients. Thus, by solving the matrix equation, Legendre and Chebychev coefficients are obtained. Some numerical examples are included to demonstrate the validity and applicability of the proposed technique.
\end{abstract}

Keywords: Volterra-Fredholm Integral Equations; Logarithmic Kernels; Integral equation; collocation matrix method; Legendre and Chebychev polynomials.

\section{Introduction}

We consider the Volterra-Fredholm integral equation of the second kind with Logarithmic Kernel:

$\psi(x, t)-\int_{0}^{t} \int_{-1}^{1} \ln |x-y| \psi(y, \tau) d y d \tau=f(x, t),(x, t) \in[-1,1] \times[0, T]$

where $0 \leqslant T$ and $f$ is a given function. The elements $\mathcal{K}(x, y)=\ln |x-y|$ is Logarithm Kernel.

For solving Volterra-Fredholm integral equations, many methods with enough accuracy and efficiency have been used before by many researches $[4,5,6,7,8,9,10,11,12]$. Maleknejad and Fadaei Yami [8] solved the system of Volterra-Fredholm integral equations by Adomian decomposition method. Kauthen in [7], used continuous time collocation method for Volterra-Fredholm integral equations. Legendre wavelets also were applied for solving Volterra-Fredholm integral equations [13]. In [14], Yalsinbas developed numerical solution of nonlinear VolterraFredholm integral equations by using Taylor polynomials. In this paper, we use numerical technique based on 
Trapezoidal rule, to reduce the Volterra-Fredholm integral Equations to a linear system of Fredholm Integral equations which will be solved using Legendre, Chebyshev collocation method (this technique is presented for the Abel Kernel in [1,2]) and new technique based on smooth transformation. The paper is organized as follows. In section 2, a system of Fredholm integral equations of the second kind is obtained from the Volterra-Fredholm Integral equation. In Sections 3 and 4, we present the Legendre and Chebychev collocation Method to solve the system obtained with special choice of the nodes. In section 5, we derive a smooth transformation to cancel the singularities in the kernel. In the remainder of the paper, we give a practical example to certify the validity of the proposed technique.

\section{System of Fredholm equations}

We consider the Volterra-Fredholm integral equation of the second kind with Logarithm Kernel (1). First, if $t=0$ the Volterra-Fredholm integral equations is reduced to: $\psi(x, 0)=f(x, 0)$. For $t \neq 0$, we apply Trapezoidal Method to solve the Volterra integral equations according to the variable $\tau$. For a given $t$, we divide the interval of integration $(0 ; t)$ into $m$ equal subintervals, $\delta \tau=\frac{t_{m}-0}{m}$, where $t_{m}=t$. Let $\tau_{0}=0, t_{0}=\tau_{0}, t_{m}=\tau_{m}=t, \tau_{j}=j \delta \tau, t_{j}=\tau_{j}$. Using the trapezoid rule,

$$
\int_{0}^{t} \int_{-1}^{1} \ln |x-y| \psi(y, \tau) d y d \tau \sim \delta \tau \sum_{j=0}^{m} \int_{-1}^{1} \ln |x-y| \psi\left(y, \tau_{j}\right) d y
$$

where the double prime indicates that the first and last term to be halved, where

$$
\delta \tau=\frac{\tau_{j}-0}{j}=\frac{t-0}{m}, \tau_{j} \leqslant t, j \geqslant 1, t=t_{m}=\tau_{m}
$$

In all our approximation, the error assumed negligible, this help us to get a system of Fredholm Integral equations.

Now, for $0 \leqslant r \leqslant m$, the Volterra Fredholm integral equations become a system of Fredholm integral equations

$$
\psi\left(x, t_{r}\right)-\delta \tau \sum_{j=0}^{r} \int_{-1}^{1} \ln |x-y| \psi\left(y, \tau_{j}\right) d y=f\left(x, t_{r}\right), 1 \leqslant r
$$

and $\psi(x, 0)=f(x, 0)$. We get the system:

$$
\begin{aligned}
& \psi(x, 0)=f(x, 0) \\
& \psi\left(x, t_{1}\right)-\frac{\delta \tau}{2} \int_{-1}^{1} \ln |x-y| \psi\left(y, t_{1}\right) d y=f\left(x, t_{1}\right)+\frac{\delta \tau}{2} \int_{-1}^{1} \ln |x-y| \psi(y, 0) d y \\
& \psi\left(x, t_{2}\right)-\frac{\delta \tau}{2} \int_{-1}^{1} \ln |x-y| \psi\left(y, t_{2}\right) d y=f\left(x, t_{2}\right)+\left\{\begin{array}{c}
\frac{\delta \tau}{2} \int_{-1}^{1} \ln |x-y| \psi(y, 0) d y \\
+\int_{-1}^{1} \ln |x-y| \psi\left(y, t_{1}\right) d y \\
\delta \tau
\end{array}\right. \\
& \psi\left(x, t_{m}\right)-\frac{\delta \tau}{2} \int_{-1}^{1} \ln |x-y| \psi\left(y, t_{m}\right) d y=f\left(x, t_{m}\right)+\delta \tau \sum_{j=0}^{m-1^{\prime}} \int_{-1}^{1} \ln |x-y| \psi\left(y, t_{j}\right) d y,
\end{aligned}
$$

where the prime indicates that the first term to be halved. Denote:

$$
f\left(x, t_{\ell}\right)=f^{\ell}(x), \psi\left(y, \tau_{\ell}\right)=\psi^{\ell}(y), \ell=0, \ldots, m
$$

Putting

$$
F^{m}(x)=f^{m}(x)+\sum_{j=0}^{m-1^{\prime}} \int_{-1}^{1} \ln |x-y| \psi^{j}(y) d y
$$


An astitute computation gives

$$
\begin{aligned}
F^{m}(x) & =f^{m}(x)+2 \sum_{j=1}^{m-1}(-1)^{j+m}\left(f^{j}(x)-\psi^{j}(x)\right) \\
& +(-1)^{m+1} \frac{\delta \tau}{2} \int_{-1}^{1} \ln |x-y| \psi^{0}(y) d y
\end{aligned}
$$

Now, our problem become:

$$
\begin{aligned}
\psi^{\ell}(x)-\frac{\delta \tau}{2} \int_{-1}^{1} \ln |x-y| \psi^{\ell}(y) d y & =F^{\ell}(x), \ell=1, \ldots, m \\
\psi(x, 0) & =f(x, 0)
\end{aligned}
$$

Equations (2) represents a system of Fredholm integral equations of the second kind. In the next, we will present the well known techniques of Legendre and Chebychev collocation methods to solve this system and later we present a smooth transformation to solve the system of Fredholm integral equation with singular kernel.

\section{Legendre collocation method}

Orthogonal polynomials are widely used in applications in mathematics, mathematical physics, engineering and computer science. One of the most common set of orthogonal polynomials is the Legendre polynomials.

We choice $x_{k}, k \in \llbracket 0, n \rrbracket$ the zeros of the Legendre polynomial of degree equal $n+1$. Here, $\llbracket a, b \rrbracket$ used to indicate the interval of all integers between $a$ and $b$. We determine a suitable interpolating elements $\phi_{j}(x), j=0,1, \ldots, n$, such that

$\psi_{n}(x)=\sum_{j=0}^{n} \phi_{j}(x) \psi\left(x_{j}\right)$

is the unique interpolating polynomial of degree $n$, which interpolates $\psi$ at the points $x_{i}, i=0,1, \ldots, n$.

The elements $\phi_{j}(x), j=0,1, \ldots, n$ are called the basic functions associated with the Legendre interpolation polynomial and they satisfy $\phi_{j}\left(x_{i}\right)=\delta_{i j}$.

Then we get an approximation of the exactly integral, let say:

$I_{n}(\psi)=\int_{-1}^{1} \mathcal{K}(x, y) \psi_{n}(y) d y$

This type of approximation must be chosen so that the integral (4) can be evaluated (either explicitly or by an efficient numerical technique).

The functions $P_{0}(x), P_{1}(x), \ldots, P_{n}(x)$ will be called interpolating elements. In this dissertation, the interpolating function $\psi_{n}$ will be assumed to be the interpolating polynomial

$\psi_{n}(x)=\sum_{j=0}^{n} \beta_{j} P_{j}(x)$

where $P_{j}$ are Legendre polynomials of degree $j, n$ is the number of Legendre polynomials, and $\beta_{j}$ are unknown parameters, to be determined.

The coefficients $\beta_{j}$ are obtained by multiplying both sides of Eq. (5) by $P_{m}, m \leqslant n$ (as weight functions), and integrating the resulting equation with respect to $x$ over the interval $[-1,1]$ to obtain

$$
\int_{-1}^{1} P_{m}(x) \psi_{n}(x) d x=\sum_{j=0}^{n} \beta_{j} \int_{-1}^{1} P_{m}(x) P_{j}(x) d x=\beta_{m} \frac{2}{2 m+1}
$$

Therefore,

$\beta_{m}=\frac{2 m+1}{2} \int_{-1}^{1} P_{m}(x) \psi_{n}(x) d x$ 
Here the integrand $P_{m} \psi_{n}$ is a polynomial of degree $n+m \leqslant 2 n$ then its integration in (6) can exactly be obtained from just $n+1$ point Gauss-Legendre method, by using the following formula

$\beta_{m}=\frac{2 m+1}{2} \sum_{j=0}^{n} w_{j} P_{m}\left(x_{j}\right) \psi\left(x_{j}\right)$

where $w_{j}, j=0, \ldots, n$ are the $(n+1)$-point Gauss-Legendre weights.

The $n+1$ grid points $\left(x_{i}\right)$ of Gauss Legendre integration in formula $(7)$ giving us the exact integral of an integrand polynomial of degree $n+m \leqslant 2 n$ can be obtained as the zeros of the $n+1$-th-degree Legendre polynomial. Then, given the $n+1$ grid point $x_{i}$, we can get the corresponding weight $w_{i}$ of the $i$ point Gauss Legendre integration formula by solving the system of linear equations. Now, the interpolating polynomial $\psi_{n}$ can be written as:

$$
\begin{aligned}
\psi_{n}(x) & =\sum_{m=0}^{n}\left(\frac{2 m+1}{2} \sum_{j=0}^{n} w_{j} P_{m}\left(x_{j}\right) \psi\left(x_{j}\right)\right) P_{m}(x) \\
& =\sum_{j=0}^{n}\left(w_{j} \sum_{m=0}^{n} \frac{2 m+1}{2} P_{m}\left(x_{j}\right) P_{m}(x)\right) \psi\left(x_{j}\right)
\end{aligned}
$$

Using (3) and (8) we get

$\phi_{j}(x)=w_{j} \sum_{m=0}^{n} \frac{2 m+1}{2} P_{m}\left(x_{j}\right) P_{m}(x), j=0, \ldots, n$

Substituting $\psi_{n}$ into Eq. (1) and collocating at the points $x_{i}$, we obtain:

$\psi\left(x_{i}\right)-\sum_{j=0}^{n} \psi\left(x_{j}\right) \int_{-1}^{1} \mathcal{K}\left(x_{i}, y\right) \phi_{j}(y) d y=f\left(x_{i}\right), i=0, \ldots, n$

To simplify the presentation let us define

$a_{i, j}=\int_{-1}^{1} \mathcal{K}\left(x_{i}, y\right) \phi_{j}(y) d y$

Then a $(n+1) \times(n+1)$ linear system is obtained:

$(I d-A) \psi=F$

where $A=\left(a_{i, j}\right)_{(i, j) \in \llbracket 0, n \rrbracket^{2}}$ is square matrix, $\psi=\left(\psi\left(x_{0}\right), \ldots, \psi\left(x_{n}\right)\right)^{t}$ and $F=\left(f\left(x_{0}\right), \ldots, f\left(x_{n}\right)\right)^{t}$, where $t$ indicate the transpose. Obviously, the system (12) has a unique solution if the determinant of the matrix $I d-A$ is nonzero, which also depends on the choice of collocation point. Substituting (9) into (11) we obtain

$$
a_{i, j}=w_{j} \sum_{k=0}^{n} \frac{2 k+1}{2} P_{k}\left(x_{j}\right) u_{k}\left(x_{i}\right)
$$

where $u_{k}\left(x_{i}\right),(i, k) \in \llbracket 0, n \rrbracket^{2}$ are defined

$$
u_{k}\left(x_{i}\right)=\int_{-1}^{1} \ln \left|x_{i}-y\right| P_{k}(y) d y
$$

The constants $u_{k}\left(x_{i}\right),(i, k) \in \llbracket 0, n \rrbracket^{2}$, can be evaluated from the recurrence relation:

$$
\begin{aligned}
(k+3) u_{k+2}\left(x_{i}\right) & =(2 k+3) x_{i} u_{k+1}\left(x_{i}\right)-k u_{k}\left(x_{i}\right), k \geqslant 1 \\
u_{0}\left(x_{i}\right) & =\left(1+x_{i}\right) \ln \left|1+x_{i}\right|+\left(1-x_{i}\right) \ln \left|1-x_{i}\right|-2 \\
u_{1}\left(x_{i}\right) & =\frac{1}{2}\left(1-x_{i}^{2}\right) \ln \left|\frac{1+x_{i}}{1-x_{i}}\right|-2 \\
u_{2}\left(x_{i}\right) & =x_{i} u_{1}\left(x_{i}\right)+\frac{2}{3}
\end{aligned}
$$




\section{Chebyshev collocation method}

Like Legendre Methods, here we will use the Chebyshev polynomials $T_{n}$ of the first kind. The polynomial $T_{n+1}$ has $n+1$ zeros in the interval $[-1 ; 1]$, which are located at the points

$x_{k}=\cos \left(\frac{2 k+1}{2 n+2} \pi\right), k \in \llbracket 0, n \rrbracket$

The Chebyshev polynomials of the first kind of degree $n, T_{n}$, satisfy discrete orthogonality relationships on the grid of the $(n+1)$ zeros of $T_{n+1}$ (which are referred to as the Chebyshev nodes):

$\sum_{k=0}^{N} T_{i}\left(x_{k}\right) T_{j}\left(x_{k}\right)= \begin{cases}0 & : i \neq j \\ N+1 & : i=j=0 \\ \frac{N+1}{2} & : i=j \neq 0\end{cases}$

For an arbitrary interval $[a, b]$, we can find a mapping that transform $[a, b]$ into $[-1,+1]$ :

$$
y_{k}=\frac{b-a}{2} x_{k}+\frac{a+b}{2}=\frac{b-a}{2} \cos \left(\frac{2 k+1}{2 n+2} \pi\right)+\frac{a+b}{2}, k \in \llbracket 0, n \rrbracket
$$

and the Chebyshev nodes defined by Eq (13) are actually zeros of this Chebyshev polynomial. Based on the discrete orthogonality relationships of the Chebyshev polynomials, various methods of solving linear and nonlinear ordinary differential equations see [3] (The solution of linear ordinary differential systems, with polynomial coefficients, can be approximated by a finite polynomial or a finite Chebyshev series. The computation can be performed so that the solution satisfies exactly a perturbed differential system, the perturbations being computed multiples of one or more Chebyshev polynomials) and integral differential equations, see [15] were devised at about the same time and were found to have considerable advantage over finite-differences methods. Since then, these methods have become standard [16]. They rely on expanding out the unknown function in a large series of Chebyshev polynomials, truncating this series, substituting the approximation in the actual equation, and determining equations for the coefficients. In our approach we follow closely the procedures like Legendre Method. Let us say, that similar procedures can be applied for a second grid given by the extremas of $T_{n}$ as nodes. It is important to stress that our goal is not to approximate a function $f$ on the interval $[-1 ; 1]$, but rather to approximate the values of the function $f$ corresponding to a given discrete set of points like those given in equation (13).

Here, let $\left(T_{0}, T_{1}, T_{2}, \ldots, T_{n}\right)$ the interpolating elements. The equation (5) becomes

$\psi_{n}(x)=\sum_{j=0}^{n '} \beta_{j} T_{j}(x)$

where the prime indicates that the first term is to be halved (which is convenient for obtaining a simple formula for all the coefficients $\beta_{j}$ ). The function $\psi_{n}$ interpolates $\psi$ at the $n+1$ Chebyshev nodes, we have at these nodes $\psi\left(x_{k}\right)=\psi_{n}\left(x_{k}\right)$. Hence, using the discrete orthogonality relation (14) we get

$$
\begin{aligned}
\beta_{j}=\frac{2}{n+1} \sum_{k=0}^{n} \psi\left(x_{k}\right) T_{j}\left(x_{k}\right), j=0,1, \ldots, n \\
\psi_{n}(x)=\sum_{j=0}^{n^{\prime}} \beta_{j} T_{j}(x) \\
=\sum_{j=0}^{n} \frac{2}{n+1} \sum_{k=0}^{n} \psi\left(x_{k}\right) T_{j}\left(x_{k}\right) T_{j}(x) \\
=\sum_{k=0}^{n} \frac{2}{n+1}\left(\sum_{j=0}^{n} T_{j}\left(x_{k}\right) T_{j}(x)\right) \psi\left(x_{k}\right)
\end{aligned}
$$


Using (3) and (17) we get:

$\phi_{k}(x)=\frac{2}{n+1} \sum_{j=0}^{n} T_{j}\left(x_{k}\right) T_{j}(x)$

Now, the same system like (12) is obtained with

$$
a_{i j}=\frac{2}{n+1} \sum_{j=0}^{n^{\prime}} v_{k}\left(x_{i}\right) T_{k}\left(x_{j}\right)
$$

where $v_{k}\left(x_{i}\right),(i, k) \in \llbracket 0, n \rrbracket^{2}$ are defined

$$
v_{k}\left(x_{i}\right)=\int_{-1}^{1} \ln \left|x_{i}-y\right| T_{k}(y) d y
$$

The constants $v_{k}\left(x_{i}\right),(i, k) \in \llbracket 0, n \rrbracket^{2}$, can be evaluated from the recurrence relation:

$$
\begin{aligned}
\left(1+\frac{1}{m+1}\right) v_{m+1}\left(x_{i}\right) & -2 x_{i} v_{m}\left(x_{i}\right)+\left(1-\frac{1}{m-1}\right) v_{m-1}\left(x_{i}\right) \\
& = \\
\frac{2}{1-m^{2}}\left(\left(1-x_{i}\right) \ln \left|1-x_{i}\right|\right. & \left.-(-1)^{m}\left(1+x_{i}\right) \ln \left|1+x_{i}\right|\right)-6 \frac{\left(1-(-1)^{m}\right)}{\left(m^{2}-1\right)\left(m^{2}-4\right)}
\end{aligned}
$$

with the starting values:

$$
\begin{aligned}
v_{0}\left(x_{i}\right) & =\left(1+x_{i}\right) \ln \left|1+x_{i}\right|+\left(1-x_{i}\right) \ln \left|1-x_{i}\right|-2 \\
v_{1}\left(x_{i}\right) & =x_{i}\left(u_{0}\left(x_{i}\right)+1\right)+\frac{1}{2}\left(\left(1-x_{i}\right)^{2} \ln \left|1-x_{i}\right|-\left(1+x_{i}\right)^{2} \ln \left|1+x_{i}\right|\right) \\
v_{2}\left(x_{i}\right) & =4 x_{i} v_{1}\left(x_{i}\right)-\left(2 x_{i}^{2}+1\right) v_{0}\left(x_{i}\right)+\frac{2}{3}\left(\left(1-x_{i}\right)^{3}+\left(1+x_{i}\right)^{3} \ln \left|1+x_{i}\right|\right)-\frac{4}{9}\left(1+3 x_{i}^{2}\right) \\
v_{3}\left(x_{i}\right) & =2 x_{i}\left(3+2 x_{i}^{2}\right) v_{0}\left(x_{i}\right)-3\left(4 x_{i}^{2}+1\right) v_{1}\left(x_{i}\right)-6 x_{i} v_{2}\left(x_{i}\right) \\
& +\left(1-x_{i}\right)^{4} \ln \left(1-x_{i}\right)+\left(1+x_{i}\right)^{4} \ln \left(1+x_{i}\right)+2 x_{i}\left(1+x_{i}^{2}\right)
\end{aligned}
$$

\section{Smoothing transformation}

Monegato and Scuderi (1998) introduce a simple smoothing change of variable to solve one-dimensional linear weakly singular integral equations on bounded intervals, with input functions which may be smooth or not. In both cases either the input function is smooth or non-smooth, they define the smoothing transformation $\kappa$ using piecewise Hermite interpolation polynomial $H_{m}$, so we will call this transformation as the Hermite transformation. The Fredholm integral equation of the second kind with logarithmic Kernel will be solved using a smmooth transformation. In our case we will present the Hermite smoothing transformation which reduce a second kind Fredholm integral equation with a weakly singular kernel, for both smooth and non-smooth input functions, to an equivalent equation with smoother solution. We choose a nonlinear transformation: $\kappa:[-1,1] \rightarrow[-1,1]$ is a sufficiently smooth monotone function having as fixed points $x_{0}=-1<x_{1}<x_{2}<\ldots<x_{n+1}=1$ and vanishing derivative at these points. An example of this mapping function is the Hermite interpolation polynomial and its is define in each subinterval by the conditions

$$
j \in\{k, k+1\}, H_{n}\left(x_{j}\right)=x_{j}, H_{n}^{(i)}\left(x_{j}\right)=0, i=0,1, \ldots, \alpha_{j}-1, \alpha_{j} \geqslant 2
$$

The integers $\alpha_{k}, k=0, \ldots, n$, are chosen accordingly to the smoothing effect that ought to produce at the points $x_{k}, k=0, \ldots, n$. Notice that the smoothness of $\kappa$ itself does not depend on the choice of $\alpha_{0}$ and $\alpha_{n}$.

A predecious choice is, $\forall k=0, \ldots, n$ :

$$
H_{k}(t)=x_{k}+\left(x_{k+1}-x_{k}\right)^{2-\alpha_{k}-\alpha_{k+1}} \frac{\left(\alpha_{k}+\alpha_{k+1}-1\right) !}{\left(\alpha_{k}-1\right) !\left(\alpha_{k+1}-1\right) !} \int_{x_{k}}^{t}\left(z-x_{k}\right)^{\alpha_{k}-1}\left(x_{k}-z\right)^{\alpha_{k+1}-1} d z .
$$

Now, using the Hermite interpolation polynomial to solve the Fredholm integral equation (in our case is nothing but the system of integral equation).

Fixing $\ell \in\{1, \ldots, m\}$ and putting $x=\kappa(t)=H_{n}(t)$ in the following system of Fredholm integral equation 
$\psi^{\ell}\left(H_{m}(t)\right)-\frac{\delta \tau}{2} \int_{-1}^{1} \ln \left|H_{m}(t)-y\right| \psi^{\ell}(y) d y=F^{\ell}\left(H_{m}(t)\right)$

a simple change of variable $y=H_{m}(s)$, then (19) becomes:

$\psi^{\ell}\left(H_{m}(t)\right)-\frac{\delta \tau}{2} \int_{-1}^{1} \ln \left|H_{m}(t)-H_{m}(s)\right| \psi^{\ell}\left(H_{m}(s)\right) H_{m}^{\prime}(s) d s=F^{\ell}\left(H_{m}(t)\right)$

Multiplying both sides of (20) by $H_{m}^{\prime}(t)$ and setting $\psi^{\ell}\left(H_{m}(t)\right) H_{m}^{\prime}(t)=\eta^{\ell}(t)$ and $F^{\ell}\left(H_{m}(t)\right) H_{m}^{\prime}(t)=\zeta^{\ell}(t)$ we obtain:

$$
\eta^{\ell}(t)-\frac{\delta \tau}{2} \int_{-1}^{1} \ln \left|H_{m}(t)-H_{m}(s)\right| H_{m}^{\prime}(t) \eta^{\ell}(s) d s=\zeta^{\ell}(t)
$$

Using

$$
\ln \left|H_{m}(t)-H_{m}(s)\right|=\ln \left|\frac{H_{m}(t)-H_{m}(s)}{t-s}\right|+\ln |t-s|
$$

for simplicity we define:

$$
\theta(t, s)=\left\{\begin{array}{r}
\ln \left|\frac{H_{m}(t)-H_{m}(s)}{t-s}\right| H_{m}^{\prime}(t), \text { if } t \neq s \\
\ln \left|H_{m}^{\prime}(t)\right| H_{m}^{\prime}(t), \text { if } t=s
\end{array}\right.
$$

Putting $t=x_{i}, i=1, \ldots, n$

$\eta^{\ell}\left(x_{i}\right)-\frac{\delta \tau}{2} \int_{-1}^{1}\left(\theta\left(x_{i}, s\right)+\ln \left|x_{i}-s\right| H_{m}^{\prime}\left(x_{i}\right)\right) \eta^{\ell}(s) d s=\zeta^{\ell}\left(x_{i}\right)$

The function $H_{m}^{\prime}\left(x_{i}\right) \eta^{\ell}(s)$ and $\theta\left(x_{i}, s\right) \eta^{\ell}(s)$ are continuous as functions of $s$,

Now we will approximate the function $\chi_{1}^{\ell}(s)=H_{m}^{\prime}\left(x_{i}\right) \eta^{\ell}(s)$ and $\chi_{2}^{\ell}(s)=\theta\left(x_{i}, s\right) \eta^{\ell}(s)$ by the $n^{\text {th }}$ degree interpolating polynomials

$\chi_{1}^{\ell}(s)=\sum_{j=0}^{n} H_{m}^{\prime}\left(x_{i}\right) \eta^{\ell}\left(x_{j}\right) \phi_{j}(s), \chi_{2}^{\ell}(s)=\sum_{j=0}^{n} \theta\left(x_{i}, x_{j}\right) \eta^{\ell}\left(x_{j}\right) \phi_{j}(s)$

which interpolates $\chi_{1}^{\ell}(s)$ and $\chi_{2}^{\ell}(s)$ at $x_{i}, i=1, \ldots, n$ and $\phi_{j}(s)$ is given by (9) (if we use Legendre polynomial) and (18) (for Chebyshev polynomial). Substituting (22) into (21) we get:

$$
\eta^{\ell}\left(x_{i}\right)-\frac{\delta \tau}{2} \sum_{j=0}^{n}\left(\chi_{1}^{\ell}\left(x_{j}\right) \int_{-1}^{1} \phi_{j}(s) d s+\chi_{2}^{\ell}\left(x_{j}\right) \int_{-1}^{1} \phi_{j}(s) \ln \left|x_{i}-s\right| d s\right) \eta^{\ell}\left(x_{j}\right)=\zeta^{\ell}\left(x_{i}\right), i=1, \ldots, n
$$

Equation $(23)$ can be written as the $(n+1) \times(n+1)$ linear system

$$
\left(I d-\frac{\delta \tau}{2} A\right) \eta^{\ell}=\zeta^{\ell}
$$

where

$$
\begin{aligned}
\eta^{\ell} & =\left(\eta^{\ell}\left(x_{i}\right)\right)_{i=1, \ldots, n}, \quad \zeta^{\ell}=\left(\zeta^{\ell}\left(x_{i}\right)\right)_{i=1, \ldots, n} \\
A & =\left(A_{i, j}=\left(\chi_{1}^{\ell}\left(x_{j}\right) \int_{-1}^{1} \phi_{j}(s) d s+\chi_{2}^{\ell}\left(x_{j}\right) \int_{-1}^{1} \phi_{j}(s) \ln \left|x_{i}-s\right| d s\right)\right)_{i, j=1, \ldots, n}
\end{aligned}
$$


The coefficient $\int_{-1}^{1} \phi_{j}(s) \ln \left|x_{i}-s\right| d s$ used in the matrix $A$ can be computed using the sections 3 and 4 while the second coefficient $\int_{-1}^{1} \phi_{j}(s) d s$ can be computed as follows:

1. Using Legendre collocation method: substituting (9) into $\int_{-1}^{1} \phi_{j}(s) d s$ and a simple computation gives

$$
\int_{-1}^{1} P_{m}(x) d x= \begin{cases}2 & \text { if } m=0 \\ 0 & \text { if } m \geqslant 1\end{cases}
$$

Now we get

$$
\int_{-1}^{1} \phi_{j}(s) d s=w_{j} \sum_{m=0}^{n} \frac{2 m+1}{2} P_{m}\left(x_{j}\right) \int_{-1}^{1} P_{m}(x) d x=w_{j}
$$

2. Using Chebychev collocation method: substituting (18) into $\int_{-1}^{1} \phi_{j}(s) d s$ and simple computation gives

$$
\int_{-1}^{1} T_{m}(x) d x=\left\{\begin{aligned}
\frac{2}{1-m^{2}} & \text { if } m \text { even } \\
0 & \text { if } m \text { odd }
\end{aligned}\right.
$$

In this case we have

$$
\begin{aligned}
\int_{-1}^{1} \phi_{j}(s) d s & =\frac{2}{n+1} \sum_{j=0}^{n}{ }^{\prime} T_{j}\left(x_{k}\right) \int_{-1}^{1} T_{j}(s) d s \\
& =\frac{2}{n+1} \sum_{j=0}^{\lfloor n / 2\rfloor^{\prime}} T_{2 j}\left(x_{k}\right) \frac{2}{1-4 j^{2}} \\
& =\frac{2}{n+1} \sum_{j=0}^{\lfloor n / 2\rfloor} \cos \left(\frac{j \pi(2 k+1)}{n+1}\right) \frac{2}{1-4 j^{2}}
\end{aligned}
$$

where $\lfloor n / 2\rfloor$ is the greatest integer less than or equal to $n / 2$.

\section{Numerical Implementation}

In this section, to achieve the validity, the accuracy and support our theoretical discussion of the proposed method, we give some computational results. The computations, associated with the example, are performed by MATLAB 7. Practically, the error function en at step $n$ is computed as $\psi^{N}-\psi_{n}$ where un is the result with $n$ used in the approximation and $N$ is large enough so that $\psi^{N}$ is much closer to the (discrete) solution $\eta^{\infty}$ than the numerical tolerance $\epsilon=10^{-12}$. We consider the solution with $n=128$, and we compute the error between the solution for different value at different value of $n$ and the $\eta^{128}$. In our computation, we consider $f(x)=x$. The method of smoothing the kernel used to solve Volterra-Fredholm integral equation with:

- Legendre approximation with $\alpha_{0}=\alpha_{1}=2$, we get Fig. 1. It's clear that the error is deceasing function for different value of $t$. We call this method "LASK".

- Chebychev approximation, with $\alpha_{0}=\alpha_{1}=2$, we get Fig. 1. It's clear that the error is deceasing function for different value of $t$. We call this method "CASK".

- Table 1 shows the $\left\|\psi-\psi_{n}\right\|_{\infty}$ with respect the variation of $n$ (number of term used in our approximation). 

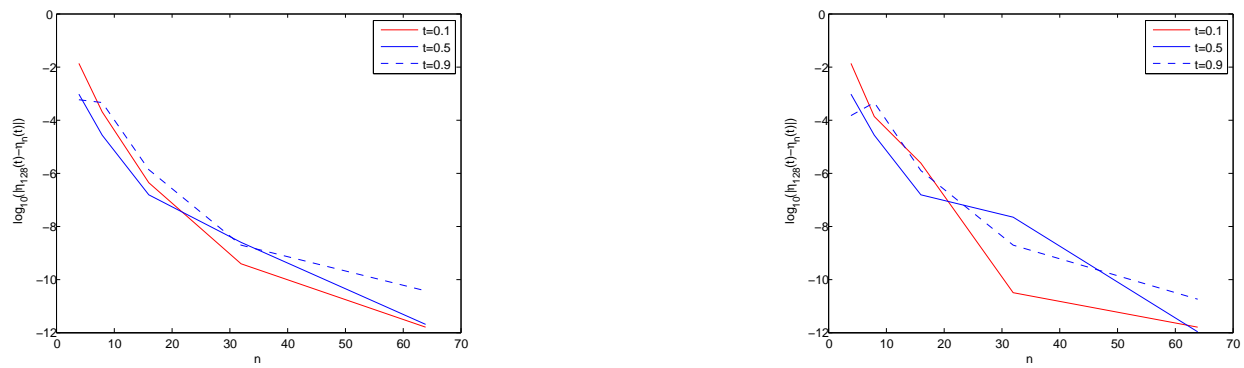

Figure 1: LASK (left figure) and CASK (right figure)

Table 1: Infinity norm of the error for different value of $n$.

\begin{tabular}{ccc}
\hline$n$ & $\left\|\psi-\psi_{n}\right\|_{\infty, L A S K}$ & $\left\|\psi-\psi_{n}\right\|_{\infty, C A S K}$ \\
\hline 32 & $1.8625 \mathrm{e}-2$ & $2.7312 \mathrm{e}-2$ \\
\hline 64 & $3.3217 \mathrm{e}-3$ & $2.4245 \mathrm{e}-3$ \\
\hline 128 & $5.5560 \mathrm{e}-4$ & $3.771 \mathrm{e}-4$ \\
\hline 256 & $8.9868 \mathrm{e}-5$ & $7.019 \mathrm{e}-5$ \\
\hline
\end{tabular}

\section{Conlusion}

- An advantage of the integration method is that it can be used to calculate integrals with singularities.

- The Legendre and Chebychev polynomials basis has been developed to solve singular Fredholm integral equations.

- Numerical results have been obtained with great accuracy.

- This method may be applied to solve Volterra Fredholm integral equations with singular Kernels and a nonlinear Volterra integral equation.

- Other type of singular Kernels can be investigate using the same method.

\section{References}

[1] C.Chniti, Numerical Approximations of Fredholm Integral equations with Abel Kernel using Legendre and Chebychev Polynomials, J. Math. Comput. Sci. 3 (2013), No. 2, 655-667.

[2] C. Chniti, On The Numerical Solution of Volterra-Fredholm Integral Equations With Abel Kernel Using Legendre Polynomials, IJAST, Issue 3 volume 1,(2013), 404-412.

[3] L. Fox, Chebyshev Methods for Ordinary Differential Equations, Comput Journal, 4, 318-331 (1962)

[4] R. Ezzati, F. Mokhtari, Numerical solution of Fredholm integral equations of the second kind by using fuzzy transforms, International Journal of Physical Science, 7 (2012) 1578- 1583.

[5] R. Ezzati, S. Najafalizadeh, Numerical solution of nonlinear Voltra-Fredholm integral equation by using Chebyshev polynomials, Mathematical Sciences Quarterly Journal, 5 (2011) 14-22. 
[6] H. Guoqiang, Z. Liqing, Asymptotic expantion for the trapezoidal Nystrom method of linear Voltra-Fredholm integral equations, J Comput Math. Appl 51 (1994) 339-348.

[7] PG. Kauthen, Continuous time collocation methods for Voltra-Fredholm integral equations, Numer Math 56 (1989) 409-424.

[8] K. Maleknejad, MR. Fadaei Yami, A computational method for system of Volterra- Fredholm integral equations, Appl. Math. Comput, 188 (2006) 589-595.

[9] K. Maleknejad, M. Hadizadeh, A new computational method for Volterra-Fredholm integral equation, J. Comput. Math. Appl 37 (1999) 37-48.

[10] K. Maleknejad, Y. Mahmodi, Taylor polynomial solution of high-order nonlinear Volterra-Fredholm integro-differential equations, Appl. Math. Comput, 145 (2003) 641-653.

[11] K. Maleknejad, S. Sohrabi. Y. Rostami, Numerical solution of nonlinear Voltra integral equation of the second kind by using Chebyshev polynomials, Appl. Math. Comput, 188 (2007) 123-128.

[12] K. Maleknejad, M. Tavassoli, Y. Mahmoudi, Numerical solution of linear Fredholm and Volterra integral equations of the second kind by using Legendre wavelet, J. Syst. Math, 32 (2003) 1530-1539.

[13] S. Yousefi, S. M. Razzaghi, Legendre wavelets method for the nonlinear Volterra- Fredholm integral equations, Math. Comput. Simul 70 (2005) 1-8.

[14] S. Yalsinbas, Taylor polynomial solution of nonlinear Volterra-Fredholm integral equations, Appl. Math. Comput, 127 (2002) 195-206.

[15] David Elliott, Chebyshev series method for the numerical solution of Fredholm integral equations. Comput. J, 6 (1963/1964), 102-111.

[16] W. H. Press, S. A. Teukolsky, W. T. Vetterling, B. P. Flannery, Numerical Recipes in FORTRAN: the art of scientific computing, Cambridge University Press, New York (1992). 